\title{
High-resolution neutron spectroscopy on protein solution samples
}

\author{
Marco Grimaldo ${ }^{1,2}$, Felix Roosen-Runge ${ }^{1}$, Niina Jalarvo ${ }^{3,4}$, Michaela Zamponi ${ }^{3}$, Fabio Zanini ${ }^{2,5}$, Marcus Hennig ${ }^{1,2}$, \\ Fajun Zhang ${ }^{2}$, Frank Schreiber ${ }^{2}$ and Tilo Seydel ${ }^{1, \text { a }}$ \\ ${ }^{1}$ Institut Max von Laue - Paul Langevin (ILL), CS20156, 38042 Grenoble, France \\ ${ }^{2}$ Institut für Angewandte Physik, University of Tübingen, 72076 Tübingen, Germany \\ ${ }^{3}$ Jülich Centre for Neutron Science JCNS, Forschungszentrum Jülich GmbH, 52425 Jülich, Germany \\ ${ }^{4}$ JCNS Outstation at the Spallation Neutron Source, Oak Ridge National Laboratory, Oak Ridge, TN 37831-6475, USA \\ ${ }^{5}$ Max-Planck Institute for Developmental Biology, Spemannstraße 35, 72076 Tübingen, Germany
}

\begin{abstract}
Proteins in solution move subject to a complex superposition of global translational and rotational diffusion as well as internal relaxations covering a wide range of time scales. With the advent of new high-flux neutron spectrometers in combination with enhanced analysis frameworks it has become possible to separate these different contributions. We discuss new approaches to the analysis by presenting example spectra and fits from data recorded on the backscattering spectrometers IN16, IN16B, and BASIS on the same protein solution sample. We illustrate the separation of the rotational and translational diffusion contribution, the accurate treatment of the solvent contribution, and the extraction of information on internal fluctuations. We also exemplify the progress made in passing from second- to third-generation backscattering spectrometers.
\end{abstract}

\section{Introduction}

The relaxational dynamics of hydrated proteins on nanometer length scales has been investigated in great depth using incoherent neutron spectroscopy due to the fundamental biological interest of dynamics-function relationships [1-5]. With the advent of high-flux neutron sources and optimized spectrometers, during the last approximately 15 years it has become possible to also study proteins in aqueous solution, where the internal fluctuations are superimposed by the global diffusive motions of the proteins [6-10]. Recent interest has focused on quantitatively understanding the global translational diffusion of the proteins in aqueous solutions [8] in terms of models derived from colloid physics [11,12]. Here, incoherent neutron spectroscopy can unambiguously access the self-diffusion and, thus, provide unique information on hydrodynamic interactions [8].

A series of challenges arises in this context when interpreting the superimposed signals from the different contributions to the recorded scattering intensity, which is dominated by the incoherent scattering of the hydrogen protons of the protonated proteins when these are suspended in heavy water $\left(\mathrm{D}_{2} \mathrm{O}\right)$ solutions. At physiological temperatures, using the relatively narrow dynamic range of the backscattering spectrometers IN10 [13] and IN16 [14] (ILL, Grenoble) (approximately $-15 \mu \mathrm{eV}<\hbar \omega<+15 \mu \mathrm{eV}$ in energy $\hbar \omega$ at a resolution of $0.9 \mu \mathrm{eV}$ FWHM), it has been possible to reliably approximate the contribution from the $\mathrm{D}_{2} \mathrm{O}$ solvent as an apparent constant background. Furthermore, the contribution from the internal diffusive fluctuations of the proteins on the nanosecond time and nanometer length scale accessible by these high-resolution spectrometers

\footnotetext{
a e-mail: seydel@ill.eu
}

could be well described by a Lorentzian line shape with a fixed width not depending on the scattering vector $q$ [8]. The subsequent steps in the analysis have in this case been the separation of the rotational $D_{r}$ and translational $D_{t}$ contributions to the apparent global diffusion coefficient $D(\varphi)=D\left(D_{r}(\varphi), D_{t}(\varphi)\right)$ of the proteins and the precise modeling of the protein shape to accurately obtain the protein volume fraction $\varphi$ occupied by the dissolved proteins. When the dynamic range of the spectrometer is increased, e.g. to $-30 \mu \mathrm{eV}<\hbar \omega<+30 \mu \mathrm{eV}$ using IN16B (ILL, Grenoble), or at a relaxed resolution to $-100 \mu \mathrm{eV}<\hbar \omega<+100 \mu \mathrm{eV}$ using BASIS (SNS, Oak Ridge), the situation becomes more complex, and the obtained information more rich. In this case, both the $\mathrm{D}_{2} \mathrm{O}$ solvent diffusion and the internal protein fluctuations contribute in a non-negligible non-constant manner to the measured signal, depending on $q$ and $\omega$.

In this article, we elucidate the essential analysis steps in detail, which are a prerequisite for understanding protein solution data. We put a particular emphasis on the recent progress in the analysis frameworks. In the following section, we briefly compare the spectrometers IN16, IN16B, and BASIS in the context of protein solution data. Subsequently, we explain a new procedure to treat the contribution of the $\mathrm{D}_{2} \mathrm{O}$ solvent. Finally, we review the separation of $D_{r}$ and $D_{t}$ and show that in the scattering vector range accessed by IN16, IN16B, and BASIS the rotational contribution does not depend on $q$ for typical proteins.

\section{New possibilities with high-flux spectrometers}

Very recently, significant progress has been made with the advent of new cold neutron backscattering spectrometers.

This is an Open Access article distributed under the terms of the Creative Commons Attribution License 4.0, which permits unrestricted use, distribution, and reproduction in any medium, provided the original work is properly cited. 
Amongst those, the most recently commissioned IN16B impresses with its significantly enhanced flux at the sample compared to its predecessor instrument IN16 (Fig. 1, top), both using the same reactor neutron source. The flux increase in this case has been achieved solely by fundamentally redesigning the neutron optics [15] based amongst other concepts on the phase space transformation [16], since the source flux remains constant.

The scattering function obtained from protonated proteins in aqueous $\left(\mathrm{D}_{2} \mathrm{O}\right)$ solution using a high-resolution neutron spectrometer can be modeled as

$$
\begin{aligned}
S(q, \omega)= & \mathscr{R} \otimes\left\{\beta \left[A_{0}(q) \mathscr{L}(\gamma, \omega) \ldots\right.\right. \\
& \left.+\left(1-A_{0}(q)\right) \mathscr{L}(\gamma+\Gamma, \omega)\right] \ldots \\
& \left.+\beta_{\mathrm{D}_{2} \mathrm{O}} \mathscr{L}\left(\gamma_{\mathrm{D}_{2} \mathrm{O}}, \omega\right)\right\} .
\end{aligned}
$$

Therein, $\mathscr{R}$ denotes the energy resolution function and $\mathscr{L}(\ldots, \omega)$ stand for Lorentzian functions with the respective linewidth given by the first parameter associated with different contributions from the sample. $\gamma$ describes the global diffusion of the proteins, consisting of both translational and rotational contributions. $\Gamma$ represents the internal relaxations accessed by the dynamic window of the respective spectrometer, and $\gamma_{\mathrm{D}_{2} \mathrm{O}}$ the contribution of the solvent. $\beta, \beta_{\mathrm{D}_{2} \mathrm{O}}$, and $A_{0}$ are scalars, and $A_{0}=A_{0}(q)$ can be identified with the elastic incoherent structure factor (EISF) which contains information on the geometry of confinement of the internal fluctuations $[17,18]$.

Example spectra (symbols) recorded on IN16 [14], IN16B [15], and BASIS [19] are depicted in Fig. 1 (top and bottom, respectively). In all experiments, the samples were held in identical double-walled cylindrical Al cells with outer radius $23 \mathrm{~mm}$ and annular gap $0.15 \mathrm{~mm}$. Please note that the statistical errors of the IN16B and BASIS spectra do not allow for a direct comparison, because the channel width in energy as well as the flux per energy interval are different, given the different design principles of these instruments. Furthermore, we note that the fast solvent water contributes differently to the signal and errors on all three spectrometers due to the different dynamic ranges. The three Lorentzians included in Eq. (1) are denoted by dashed, dash-dotted, and solid lines lines, respectively. BASIS achieves an energy resolution of approximately $3.5 \mu \mathrm{eV}$ FWHM. By contrast, IN16B [15] operates in exact backscattering and therefore achieves a higher energy resolution of $0.9 \mu \mathrm{eV}$ FWHM. Also on IN16B, which covers a smaller energy range associated with the higher energy resolution compared to BASIS, a significant energy-dependent contribution of the $\mathrm{D}_{2} \mathrm{O}$ solvent is visible.

Importantly, given the good quality of the data that can now be obtained, the spectra at the individual scattering vectors can be fitted by Eq. (1) with free parameters $0 \leq \beta \leq \infty, 0 \leq A_{0} \leq 1,0 \leq \gamma \leq \infty$, and $0 \leq \Gamma \leq \infty$. The prerequisite for such a fit without imposing further constraints is an accurate description of the solvent contribution. In this way, $\beta_{\mathrm{D}_{2} \mathrm{O}}$ and $\gamma_{\mathrm{D}_{2} \mathrm{O}}$ can be fixed, as explained in the following section. Further, the resolution function $\mathscr{R}=\mathscr{R}(\omega)$ has to be accurately taken into account by describing it analytically as a sum of Gaussian functions. In the numerical implementation of the fit, the
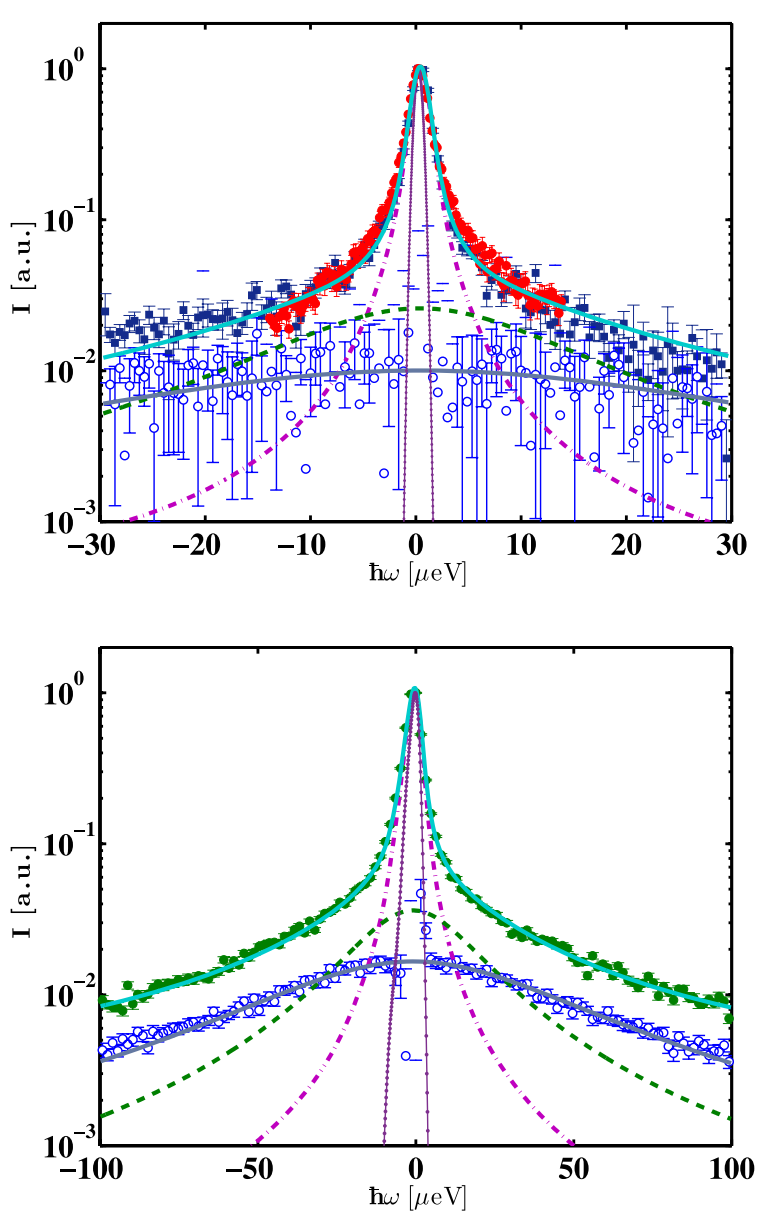

Figure 1. Top: comparison of spectra recorded using the former spectrometer IN16 (red circles, narrow spectrum, 256 channels with width $0.12 \mu \mathrm{eV}$ ) and the new spectrometer IN16B (blue squares, broad spectrum, 256 channels with width $0.24 \mu \mathrm{eV}$ ) on BSA proteins in $\mathrm{D}_{2} \mathrm{O}$ solution (same BSA concentration $200 \mathrm{mg} / \mathrm{ml}$ and $T=295 \mathrm{~K}$ for both spectra) at the same scattering vector $q=0.6 \AA^{-1}$, subsequent to the empty can subtraction, illustrating the progress made with IN16B. The IN16 data have been recorded during 24 hours, the IN16B data during only 4 hours. The solid line superimposed on the data denotes the fit to the IN16B data according to Eq. (1) and the dashed and dash-dotted lines denote the Lorentzians $\mathscr{L}(\Gamma+\gamma)$ and $\mathscr{L}(\gamma)$, respectively. The narrow line with dot symbols indicates the resolution determined by a fit to a Vanadium measurement which is the same for both instruments. While on IN16 the $\mathrm{D}_{2} \mathrm{O}$-solvent contribution can be approximated by a constant background, this contribution becomes weakly $\omega$-dependent within the energy-range of IN16B (open circle symbols modeled by the Lorentzian-shaped solid line, see also section 3). All Lorentzians are displayed subsequent to the convolution with the resolution. Bottom: example spectrum (symbols) recorded on BASIS at $q=0.65 \AA^{-1}$ on a BSA solution in $\mathrm{D}_{2} \mathrm{O}$ (concentration $200 \mathrm{mg} / \mathrm{ml}, T=295 \mathrm{~K}$, counting time $\approx 4$ hours, 500 channels with width $0.4 \mu \mathrm{eV}$ ), subsequent to the empty can subtraction. The attribution of the lines is as in the top figure. The resolution function of BASIS is approximately $3.5 \mu \mathrm{eV} \mathrm{FWHM} \mathrm{wide.}$

expressions $\mathscr{R} \otimes \mathscr{L}(\ldots, \omega)$ are subsequently calculated using Voigt functions. The resolution function of IN16B can be well approximated by a single and even better by a 


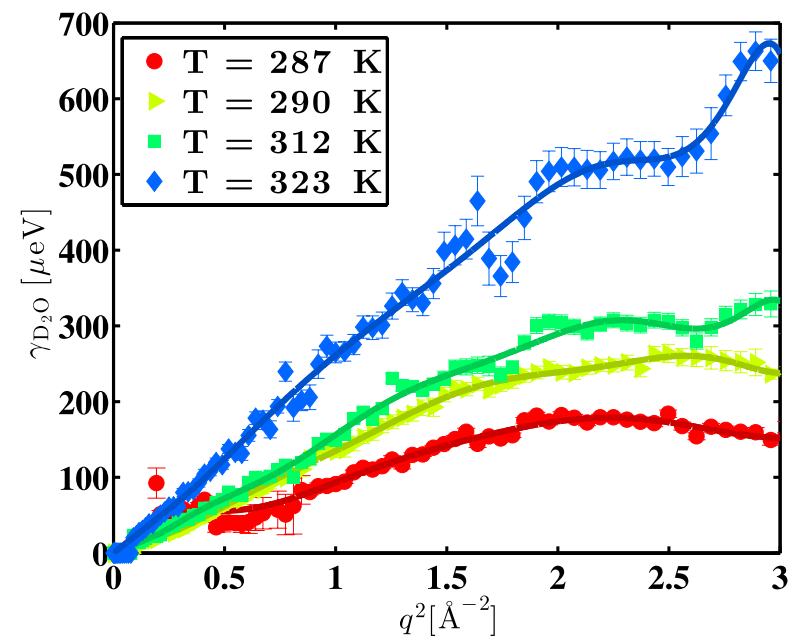

Figure 2. Linewidth $\gamma_{\mathrm{D}_{2} \mathrm{O}}$ of $\mathrm{D}_{2} \mathrm{O}$ (Eq. (2)) as a function of the scattering vector $q^{2}$ for various temperatures $T$ obtained from time-of-flight data recorded on IN6, ILL (symbols) [20], using the incident wavelength $5.1 \AA$ corresponding to a resolution of $\approx 90 \mu \mathrm{eV}$ FWHM. The lines are polynomial fits. Note that at the lowest temperature, the fit becomes unstable for $q^{2}<0.5 \AA^{-2}$. (Figure reprinted with permission from the supplementary material of Ref. [18].)

sum of two Gaussians centered at $\omega=0$ (the latter is the case for the fits in Fig. 1, top). The resolution of BASIS is modeled by a sum of 4 Gaussians centered at $\omega \neq 0$ (Fig. 1, bottom).

\section{The contribution from the solvent water}

The scattering function of the solvent water can be modeled as [21]

$$
S_{\mathrm{D}_{2} \mathrm{O}}(q, \omega)=A_{\gamma} \mathscr{L}\left(\gamma_{\mathrm{D}_{2} \mathrm{O}}, \omega\right)+A_{\Gamma} \mathscr{L}\left(\Gamma_{\mathrm{D}_{2} \mathrm{O}}, \omega\right)+B(q),
$$

where $\mathscr{L}\left(\gamma_{\mathrm{D}_{2} \mathrm{O}}, \omega\right)$ denotes the Lorentzian function describing the dynamics of the entire water molecule, and $\mathscr{L}\left(\Gamma_{\mathrm{D}_{2} \mathrm{O}}, \omega\right)$ accounts for faster movements, the width $\Gamma_{\mathrm{D}_{2} \mathrm{O}}$ being a factor 3 or more larger than $\gamma_{\mathrm{D}_{2} \mathrm{O}}$. $A_{\gamma}$ and $A_{\Gamma}$ are scalars. $B(q)$ denotes a $q$-dependent background [21] which we find to be always smaller by at least a factor of $10^{-3}$ than the maximum of the spectrum. A slight curvature due to $\mathscr{L}\left(\gamma_{\mathrm{D}_{2} \mathrm{O}}, \omega\right)$ is detectable within the dynamic range of IN16B and BASIS (Fig. 1), whilst $\mathscr{L}\left(\Gamma_{\mathrm{D}_{2} \mathrm{O}}, \omega\right)$ is too broad to be visible on these instruments. $\gamma_{\mathrm{D}_{2} \mathrm{O}}(q, T)$ is depicted in Fig. 2, as experimentally determined using the time-of-flight spectrometer IN6. The model in Eq. (2) has originally been developed for $\mathrm{H}_{2} \mathrm{O}$. We note that due to the use of $\mathrm{D}_{2} \mathrm{O}$ as a solvent, a de Gennes-narrowing becomes visible at the highest $q$ (Fig. 2), and the $q$-dependence of the spectral intensity is modulated by the structure factor due to the coherent scattering contribution. However, since the $\mathrm{D}_{2} \mathrm{O}$-contribution to the scattering from the protein solution samples is treated individually for each $q$, the latter fact is of no importance in the present context, and the polynomial interpolation naturally also includes the modulation of the $q$-dependence of $\gamma_{\mathrm{D}_{2} \mathrm{O}}$ due to coherent scattering effects. We note that the fit results displayed in Fig. 2 may at some $q$-values and temperatures be inaccurate due to a possible cross-talking of the two Lorentzians in Eq. (2) and the resolution of IN6, as reflected by large error bars. Nevertheless, the polynomial fits over the entire $q$-range appear to be sufficiently robust. The resulting Lorentzian line shape $\mathscr{L}\left(\gamma_{\mathrm{D}_{2} \mathrm{O}}, \omega\right)$ obtained from IN6 for the solvent contribution to the protein solution spectra is therefore included in Eq. (1). The amplitude $\beta_{\mathrm{D}_{2} \mathrm{O}}$ of this contribution is determined by weighting the intensity of pure $\mathrm{D}_{2} \mathrm{O}$-spectra recorded on IN16B or BASIS, respectively, according to the $\mathrm{D}_{2} \mathrm{O}$ volume $(1-\varphi)$ in the sample,

$$
I_{\mathrm{D}_{2} \mathrm{O}}^{(\varphi)}(q, \omega)=(1-\varphi) I_{\mathrm{D}_{2} \mathrm{O}}(q, \omega) .
$$

Subsequently Eq. (2) is fitted to $I_{\mathrm{D}_{2} \mathrm{O}}^{(\varphi)}(q, \omega)$ with $\gamma_{\mathrm{D}_{2} \mathrm{O}}$ fixed from the IN6 results and $A_{\Gamma}=0$ in Eq. (2). Finally,

$$
I_{\mathrm{D}_{2} \mathrm{O}}^{(\varphi)}(q, \omega)=\beta_{\mathrm{D}_{2} \mathrm{O}} \mathscr{L}_{\mathrm{D}_{2} \mathrm{O}}\left(\Gamma_{\mathrm{D}_{2} \mathrm{O}}, \omega\right)
$$

at $\omega=0$ determines $\beta_{\mathrm{D}_{2} \mathrm{O}}$ in this fit result. In Fig. 1, we include both the water spectra measured on IN16B and BASIS, respectively (open circles), as well as the model for the $\mathrm{D}_{2} \mathrm{O}$ solvent contribution obtained from the above method (Lorentzian shaped solid line) to illustrate the validity of the method.

\section{The separation of the rotational and translational diffusion}

In Eq. (1), $\gamma$ is associated with the observable apparent diffusion coefficient $D$ via $\gamma(q)=D q^{2}$. $D$ consists of contributions from the translational diffusion $D_{t}$ and rotational diffusion $D_{r}$ through an implicit relation $D(\varphi)=D\left(D_{t}(\varphi), D_{r}(\varphi)\right)$. In general, $D>D_{t}[6]$, and $D_{r}$ thus causes an additional line broadening of $\mathscr{L}(\gamma, \omega)$ compared to a hypothetical situation without rotational diffusion (Fig. 3). The scattering function due to translational diffusion of a rigid sphere can be written as a Lorentzian with width $D_{t} q^{2}$,

$$
\mathcal{S}_{\text {inc }}^{\mathrm{T}}(q, \omega)=\mathscr{L}\left(D_{t} q^{2}, \omega\right),
$$

and the scattering function due to the rotation of a rigid sphere reads

$$
\mathcal{S}_{\text {inc }}^{\mathrm{R}}(q, \omega)=\sum_{l=0}^{\infty} B_{l}(q) \mathscr{L}\left[l(l+1) D_{r}, \omega\right],
$$

with the Lorentzian $\mathscr{L}$ with width $l(l+1) D_{r}$ and

$$
B_{l}(q)=(2 l+1) \int \rho_{H}(r) j_{l}^{2}(q r) \mathrm{d} r
$$

Therein, $j_{l}(x)$ denotes the $l$ th-order spherical Bessel function of first kind and $\rho_{H}(r)$ the hydrogen density at radius $r$. The scattering function of the superposition of the rotational and translational diffusion is the convolution

$$
\begin{aligned}
\mathcal{S}_{\mathrm{inc}}^{\mathrm{TR}}(q, \omega) & =\mathcal{S}_{\mathrm{inc}}^{\mathrm{R}}(q, \omega) \otimes \mathcal{S}_{\mathrm{inc}}^{\mathrm{T}}(q, \omega) \\
& =\sum_{l=0}^{\infty} B_{l}(q) \mathscr{L}\left(\Gamma_{l}, \omega\right)
\end{aligned}
$$



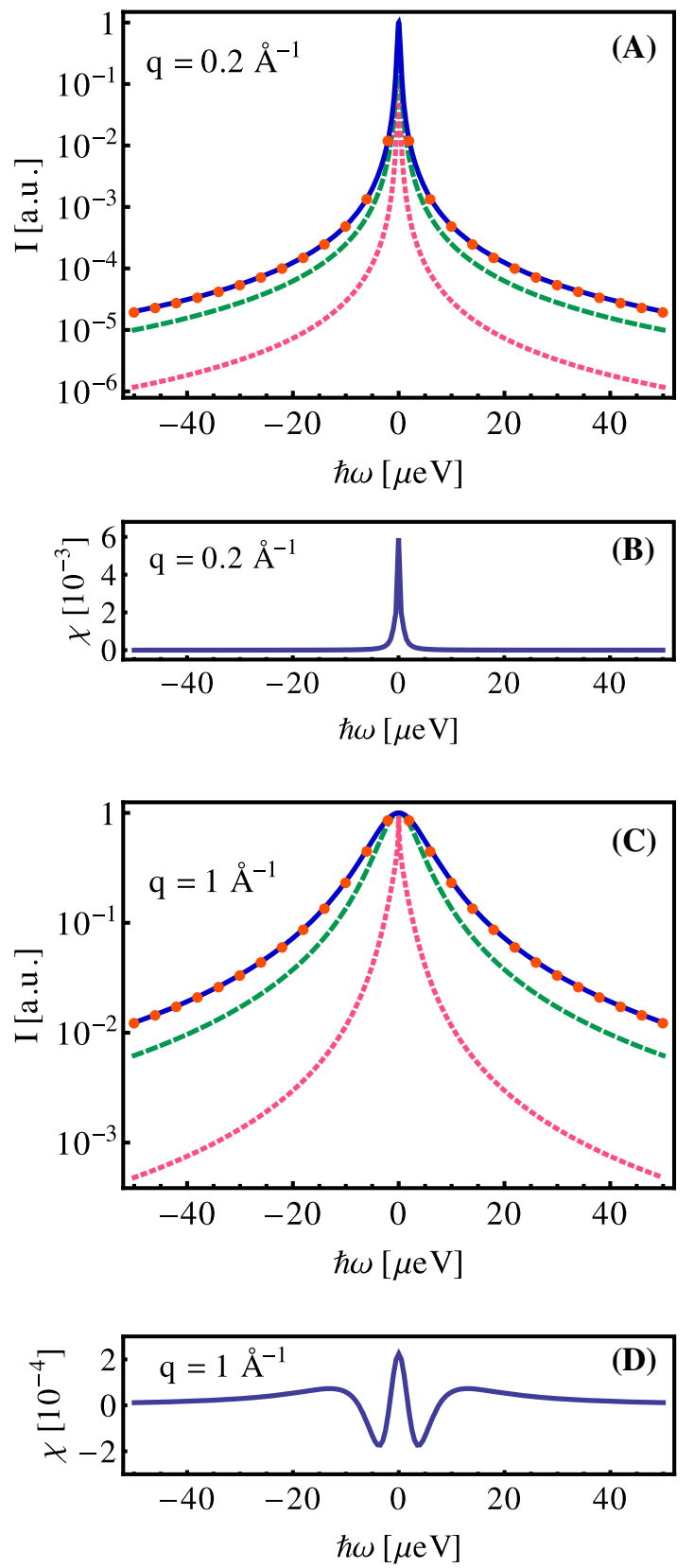

Figure 3. Parts (A) and (C): incoherent scattering function $\mathcal{S}_{\text {inc }}^{\mathrm{TR}}(q, \omega)$ of a diffusing hypothetical particle of radius $r=36 \AA$ at $q=0.2 \AA^{-1}$ (A) and $q=1 \AA^{-1}$ (C), respectively (circles). The translational and rotational diffusion coefficients are $D_{t}=$ $6 \AA^{2} / \mathrm{ns}$ and $D_{r}=3.1 \cdot 10^{-3} \mathrm{~ns}^{-1}$, respectively, for both (A) and (C), corresponding to BSA at room temperature in water. The dark blue solid line is a Lorentzian function fitted to $\mathcal{S}_{\text {inc }}^{\mathrm{TR}}(q, \omega)$. The residuals $\chi$ resulting from the fit are shown below the spectra, figure parts (B) and (D), respectively. The green dashed line and the pink short-dashed line are the incoherent scattering functions $\mathcal{S}_{\text {inc }}^{\mathrm{T}}(q, \omega)$ and $\mathcal{S}_{\text {inc }}^{\mathrm{R}}(q, \omega)$ respectively. Note that the three functions were normalized to the respective maxima. (Figure rendered using Mathematica.)

with the width $\Gamma_{l}=l(l+1) D_{r}+q^{2} D_{t}$. Perez et al. [6] have shown that the superposition of the rotational and translational diffusion of proteins in solution can be accurately described by a single Lorentzian function with
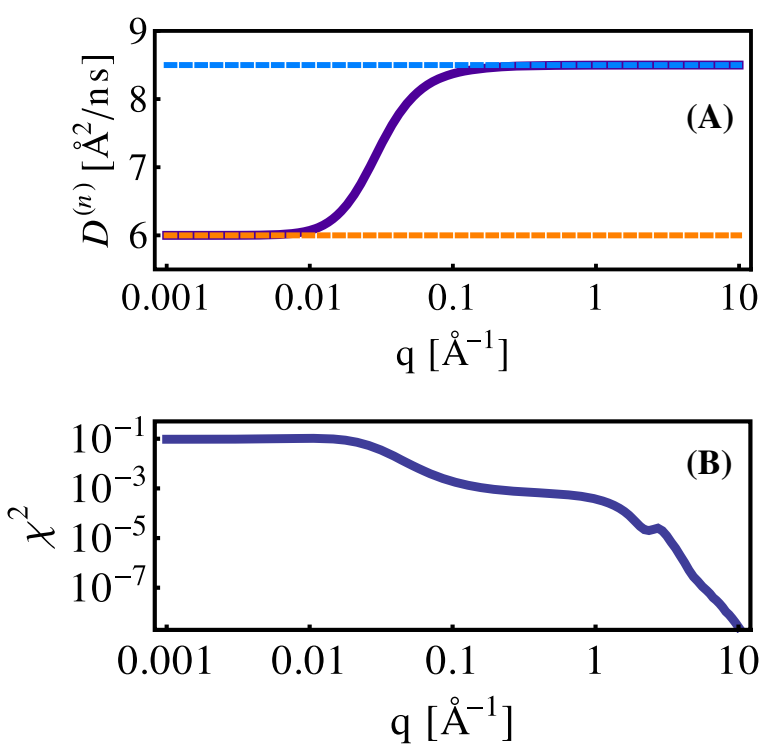

Figure 4. (A): General apparent diffusion coefficient $D^{(n)}=$ $D\left(q, D_{t}, D_{r}\right)$ of a hypothetical diffusing particle with the same parameters as in Fig. 3 (solid line). For the calculation the sum in Eq. (15) has been truncated at $n=550$, since convergence was sufficient. We observe that for $q \rightarrow 0, D \approx D_{t}$ (lower dashed line) and for $q \rightarrow \infty, D_{r}=$ const., and, thus, $D=$ const. (upper dashed line). (B): $\chi^{2}$ of the fit of a Lorentzian function to $\mathcal{S}_{\text {inc }}^{\mathrm{TR}}(q, \omega)$ as a function of $q$. (Figure rendered using Mathematica, part (A) from [22].)

the dependence $\gamma=D q^{2}$, which is in agreement with our experimental observations [8]. Therefore, the fit of this single Lorentzian to the data $\mathcal{S}_{\text {inc }}^{\mathrm{TR}}(q, \omega)$ can be written as

$$
\min _{\alpha, \gamma}\left\{\int\left[\mathcal{S}_{\text {inc }}^{\mathrm{TR}}(q, \omega)-\alpha \mathscr{L}(\gamma, \omega)\right]^{2} \mathrm{~d} \omega\right\} .
$$

Solving Eq. (9) results in two coupled nonlinear equations determining the parameters $\alpha$ and $\gamma$ [22]:

$$
\begin{aligned}
& \alpha=2 \gamma \sum_{l=0}^{\infty} \frac{B_{l}}{\Gamma_{l}+\gamma}, \\
& 0=\sum_{l=0}^{\infty} \frac{B_{l}}{\Gamma_{l}+\gamma}\left(\frac{1}{\Gamma_{l}+\gamma}-\frac{1}{2 \gamma}\right) .
\end{aligned}
$$

Assuming $\gamma=D q^{2}$ and rearranging Eq. (11) yields:

$$
\sum_{l=0}^{\infty} B_{l}(q) \frac{l(l+1) D_{r}+q^{2}\left(D_{t}-D\right)}{\left[l(l+1) D_{r}+q^{2}\left(D_{t}+D\right)\right]^{2}}=0 .
$$

We now notice that $B_{l}(q)$ strongly decays for $l>q R$, with $R=\sup \left\{r, \rho_{H}(r) \neq 0\right\}$, and therefore approximate Eq. (12) by a finite sum, thereby introducing the sequence $D^{(n)}\left(q, D_{t}, D_{r}\right)[22]:$

$$
\sum_{l=0}^{n} B_{l}(q) \frac{l(l+1) D_{r}+q^{2}\left(D_{t}-D^{(n)}\right)}{\left[l(l+1) D_{r}+q^{2}\left(D_{t}+D^{(n)}\right)\right]^{2}}=0 .
$$

Figure 3 shows $\mathcal{S}_{\text {inc }}^{\mathrm{TR}}(q, \omega)$ for a hypothetical particle of radius $r_{h}=36 \AA$ (circles) as well as the Lorentzian 
function $\mathscr{L}(\gamma, \omega)$ (dark blue solid line) and the two incoherent scattering functions $\mathcal{S}_{\text {inc }}^{\mathrm{T}}(q, \omega)$ (green dashed lines) and $\mathcal{S}_{\text {inc }}^{\mathrm{R}}(q, \omega)$ (pink short-dashed lines) for $q=$ 0.2 and $1 \AA^{-1}$. Below the spectra, the residuals $\chi=$ $\mathcal{S}_{\text {inc }}^{\mathrm{TR}}(q, \omega)-\alpha \mathscr{L}(\gamma, \omega)$ of the fit in Eq. (9) as a function of $\hbar \omega$ confirm the goodness of the fit: a small, narrow peak centered in $\hbar \omega=0$ at $q=0.2 \AA^{-1}$ becomes a broader oscillation, but with even smaller amplitude, at $q=1 \AA^{-1}$. Also $\chi^{2}$ as a function of $q$ (Fig. 4B) corroborates the validity of the fit with a single Lorentzian function, which becomes increasingly precise at higher q. $D^{(n)}\left(q, D_{t}, D_{r}\right)$ obtained from Eq. (13) with $n=550$ for the same particle as before is shown in Fig. 4A. We observe that $D^{(n)}\left(q, D_{t}, D_{r}\right)$ starts at $D_{t}$ for $q=0$ and converges rapidly to a constant value, such that in the $q$-range $0.2 \AA^{-1} \lesssim q \leq 2 \AA^{-1}$ accessible by backscattering experiments we can safely assume:

$$
D\left(D_{t}, D_{r}\right)=\lim _{n \rightarrow \infty} D^{(n)}\left(q, D_{t}, D_{r}\right)=\text { const. }
$$

Thus, Eq. (12) can be approximated by

$$
\sum_{l=0}^{n} B_{l}(q) \frac{D_{r} l(l+1)+\left(D_{t}-D\right) q^{2}}{\left[D_{r} l(l+1)+\left(D_{t}+D\right) q^{2}\right]^{2}}=0,
$$

where $n$ must be sufficiently larger than $q R$. The sum in Eq. (15) can be truncated at $n=2 q R$, since the spherical Bessel function of first kind $j_{l}(x)$, and similarly $B_{l}(q)$, decays fast for $n>x$, as follows:

$$
j_{l}(x) \leq \sqrt{\pi} \frac{x^{l} \sqrt{\pi}}{2^{l+1} \Gamma[l+3 / 2]}<\frac{x^{l} \sqrt{\pi}}{2^{l+1} l !} .
$$

Finally, to solve the implicit relation, Eq. (15), we assume that $D_{r}$ obeys the colloid model for the rotational diffusion [23]

$$
D_{r}(\varphi)=D_{r, 0}\left(1-1.3 \varphi^{2}\right),
$$

where $D_{r, 0}$ is the rotational diffusion coefficient in the dilute limit. In this way, provided that the data and their statistics are good enough, we obtain a method to separate $D_{r}(\varphi)$ and $D_{t}(\varphi)$ from the observable quantity $D(\varphi)$ based solely on the assumption that the colloid model for the rotational diffusion is valid, which is a rather robust assumption in the short-time limit where the angular displacement of a protein is of the order of only a small fraction of a full rotation and therefore its exact shape is of minor importance. We observe that in the $q$-range covered by IN16B and BASIS, the $q$-dependence of $\mathcal{S}_{\text {inc }}^{\mathrm{TR}}(q, \omega)$ due to the rotational diffusion of a typical protein can be neglected (Fig. 4A).

\section{Conclusion and outlook}

The new high-resolution backscattering spectrometers IN16B and BASIS as well as similar recent other spectrometers such as SPHERES [24] allow for significant advances in the spectroscopy of protein solution samples. To match the progress in the neutron instrumentation, corresponding advances in the data analysis are equally important. With the present article, we have elucidated the first and fundamental steps in separating the contributions from the aqueous solvent, the rotational and translational diffusion, as well as the internal fluctuations of the proteins, using data recorded on IN16B and BASIS as examples.

We thank B. Frick, D. Bazzoli, R. Ammer, and M. Appel (ILL) for the very successful commissioning of IN16B as well as E. Mamontov (SNS) and M. Koza (ILL) for stimulating discussions. We acknowledge the allocation of beam time at the ILL and the SNS. Research conducted at ORNL's SNS was sponsored by the Scientific User Facilities Division, Office of Basic Energy Sciences, US Department of Energy. Access to BASIS was granted through the JCNS partner user program. This work was supported by the DFG.

\section{References}

[1] G. Zaccai, Science 288, 1604 (2000)

[2] F. Gabel, D. Bicout, U. Lehnert, M. Tehei, M. Weik, G. Zaccai, Q. Rev. Biophys. 35, 327 (2003)

[3] J. Pieper, A. Buchsteiner, N.A. Dencher, R.E. Lechner, T. Hauß, Phys. Rev. Lett. 100, 228103 (2008)

[4] A. Frölich, F. Gabel, M. Jasnin, U. Lehnert, D. Oesterhelt, A. Stadler, M. Tehei, M. Weik, K. Wood, G. Zaccai, Faraday Discussions 141, 117 (2009)

[5] J.F. Ankner, W.T. Heller, K.W. Herwig, F. Meilleur, D.A. Myles, in:Current Protocols in Protein Science (John Wiley \& Sons, 2013), chap. 17.16

[6] J. Perez, J. Zanotti, D. Durand, Biophys. J. 77, 454 (1999)

[7] A. Stadler, I. Digel, J. Embs, T. Unruh, M. Tehei, G. Zaccai, G. Büldt, G. Artmann, Biophys. J. 96, 5073 (2009)

[8] F. Roosen-Runge, M. Hennig, F. Zhang, R.M.J. Jacobs, M. Sztucki, H. Schober, T. Seydel, F. Schreiber, Proc. Natl. Acad. Sci. U. S. A. 108, 11815 (2011)

[9] M. Hennig, F. Roosen-Runge, F. Zhang, S. Zorn, M. Skoda, R. Jacobs, F. Schreiber, Soft Matter 8, 1628 (2012)

[10] F. Roosen-Runge, M. Hennig, T. Seydel, F. Zhang, M. Skoda, S. Zorn, R. Jacobs, M. Maccarini, P. Fouquet, F. Schreiber, BBA-Proteins Proteom. 1804, 68 (2010)

[11] M. Tokuyama, I. Oppenheim, Phys. Rev. E 50, 16 (1994)

[12] M. Heinen, F. Zanini, F. Roosen-Runge, D. Fedunova, F. Zhang, M. Hennig, T. Seydel, R. Schweins, M. Sztucki, M. Antalik et al., Soft Matter 8, 1404 (2012)

[13] O. Randl, H. Franz, T. Gerstendörfer, W. Petry, G. Vogl, A. Magerl, Physica B: Condensed Matter 234-236, 1064 (1997)

[14] B. Frick, M. Gonzalez, Physica B 301, 8 (2001)

[15] B. Frick, E. Mamontov, L.v. Eijck, T. Seydel, Z. Phys. Chem. 224, 33 (2010)

[16] M. Hennig, B. Frick, T. Seydel, J. Appl. Crystallogr. 44, 467 (2011)

[17] M. Bée, Physica B 182, 323 (1992) 
[18] M. Grimaldo, F. Roosen-Runge, F. Zhang, T. Seydel, F. Schreiber, J. Phys. Chem. B 118, 7203 (2014)

[19] E. Mamontov, K.W. Herwig, Rev. Sci. Instr. 82, 085109 (2011)

[20] The ILL Yellow Book (2008), http://www.ill. eu/fr/instruments-support/instrumentsgroups/yellowbook/

[21] J. Qvist, H. Schober, B. Halle, J. Chem. Phys. 134, 144508 (2011)
[22] M. Hennig, Ph.D. thesis, University of Tübingen (2011), http://nbn-resolving.de/urn:nbn: de: wbsz: $21-$ opus -58803

[23] A.J. Banchio, G. Nägele, J. Chem. Phys. 128, 104903 (2008)

[24] J. Wuttke, A. Budwig, M. Drochner, H. Kämmerling, F.J. Kayser, H. Kleines, V. Ossovyi, L.C. Pardo, M. Prager, D. Richter et al., Rev. Sci. Instr. 83, 075109 (2012) 